\title{
Analysis and Experiments for Dual-Rate Beacon Scheduling in ZigBee/IEEE 802.15.4
}

\author{
Shantao Chen \\ The State Key Laboratory of \\ Industrial Control Technology, \\ Zhejiang University \\ Hangzhou 310027, P.R. China \\ stchen@iipc.zju.edu.cn
}

\author{
Luís Almeida \\ Institute of Telecommunications \\ DEEC - Faculty of Engineering \\ University of Porto \\ Rua Dr. Roberto Frias, 4200-465 \\ Porto, PORTUGAL \\ lda@fe.up.pt
}

\author{
Zhi Wang \\ The State Key Laboratory of \\ Industrial Control Technology, \\ Zhejiang University \\ Hangzhou 310027, P.R. China \\ wangzhi@iipc.zju.edu.cn
}

\begin{abstract}
Cyber-Physical Networked Systems (CPNS) are systems that integrate a multitude of nodes that perform computations for the sake of interacting with, or controlling, a physical process. The network plays an important role in CPNS supporting the interactions among the distributed nodes that enable the collaborative work towards the application goals. Some systems, e.g., target tracking systems, require reconciling properties that are strongly influenced by the network in opposing directions. For example, it is important to maintain low energy consumption to prolong lifetime but it is also important to be reactive to event propagation for quick target detection. In the ZigBee/IEEE802.15.4 standards, the beacon-enabled mode can achieve low energy consumption by adopting low duty cycles but this causes reaction latency to events to degrade. In this paper, we carry out several experiments that validate the potential of the recently proposed dual-rate beacon scheduling method for ZigBee/IEEE802.15.4 sensor networks to accomplish a trade-off between low energy consumption and rapid events propagation. The experiments include both simulation with OPNET as well as a real test-bed built on CC2430-based nodes. The results show the impact of relevant configuration parameters and the superiority of the dual-rate beacon scheduling in providing low energy consumption and low latency in CPNS.
\end{abstract}

Keywords-CPNS; target tracking; ZigBee; beacon scheduling; energy consumption; transmission delay

\section{INTRODUCTION}

Cyber-Physical Network Systems (CPNS) integrate computations and communications with physical processes that impose tight temporal constraints. For example, embedded computers and networks monitor and control physical processes with feedback loops [1]. Then CPNS can expedite the connection of embedded systems and IT systems, enabling the integration of these high level systems with the physical world. To support CPNS, the network plays an important role in delivering sensing data in a reliable and timely way.

Target tracking in particular is a class of applications that utilize multiple sensors in the environment that collaborate in sensing, target detection and data fusion to provide useful information to the higher level decision-maker or automatic control system. Generally, a target tracking system is deployed in a wide field and must stay in autonomous operation for long periods. Therefore, energy conservation is of high relevance. However, when a target is detected, the system needs to react to such event rapidly to track it adequately and provide such information to the higher level, through a sink in the sensor network, in a timely way. These two desired properties depend substantially on the communication protocol that is required to satisfy both low energy consumption and low event notification latency.

Among many wireless communication protocols, ZigBee/IEEE 802.15.4[2][3] was specifically developed for sensor networks, targeting low cost, low energy consumption, high scalability, etc. It provides two alternative operation modes, namely beacon-enabled and beacon-disabled. In the former mode, the network can be operated with a low dutycycle, with long inactive periods during which nodes can enter a sleep state to save energy. On the other hand, the beacondisabled mode does not support any sleep period thus providing higher throughput without considering energy consumption. In the case of target tracking, the beacon-enabled mode can drive the network to achieve extremely low energy consumption. However, the long inactive state in beacon-enabled mode introduces potentially long transmission delays that hinder quick event propagation. In order to improve the timeliness of the beacon-enabled mode in ZigBee, reference [4] schedules the superframe of the cluster based on the routing information to decrease the transmission delay along clusters. The active period of different clusters partially overlaps if there is a routing relationship between the respective nodes, thus reducing the transmission delay between clusters. The work in [5] addressed burst traffic under IEEE 802.15.4 in a star topology with a fixed Beacon Order (BO) and Superframe Order (SO), that set the beacon interval and active period respectively, and proposed an algorithm extending the active period based on a request from devices. After the end of the active period in a superframe, the node with the burst traffic sends a busy tone to the coordinator to extend the active period to get an extra CAP (contention-access period) and thus the end-to-end delay of the burst is also reduced. The work in [6] also proposed extending the active period of the superframe duration to transmit the real-time data with Quality-of-Service guarantees. Especially, references [7][8] propose a traffic-based adaptive method to adapt the BI (Beacon Interval) to save the 
energy according to the current traffic . It changes BI based on the amount of data received by the cluster head. Several beacon interval rounds are needed to adjust the BI to a proper value. The work in [8] is an improvement to that in [7] with respect to the reaction time to increasing traffic and applied to a clustertree topology. Both of them require a long adjusting time when traffic varies.

Conversely to the other approaches, we studied the possibility of using a prompt mode-change between two very different beacon intervals that would provide different properties, a long interval for low energy consumption and a short interval for fast event propagation and then switch on-line between both depending on whether there was a target in the area of interest or not. We called our scheme dual-rate beacon scheduling, which was originally presented in [9] for improving the real-time performance of ZigBee while catering for low energy consumption. In this work, we complement the work in [9] with a comprehensive assessment of the impact on performance that protocol parameters have and we show the experimental results obtained with a test-bed based on the CC2430 network controller. These results highlight the benefits of the proposed method and its applicability to target tracking systems and CPNS in general.

The rest of this paper is organized as follows: Section 2, briefly introduces our beacon scheduling method. Section 3 describes the impact of various parameters on the performance of our method. Section 4 introduces our test-bed and the experiment results. Finally, Section 5 concludes the paper.

\section{BEACON SCHEDULING METHOD}

\section{A. Scheduling Method}

Given a ZigBee network as in Fig. 1:

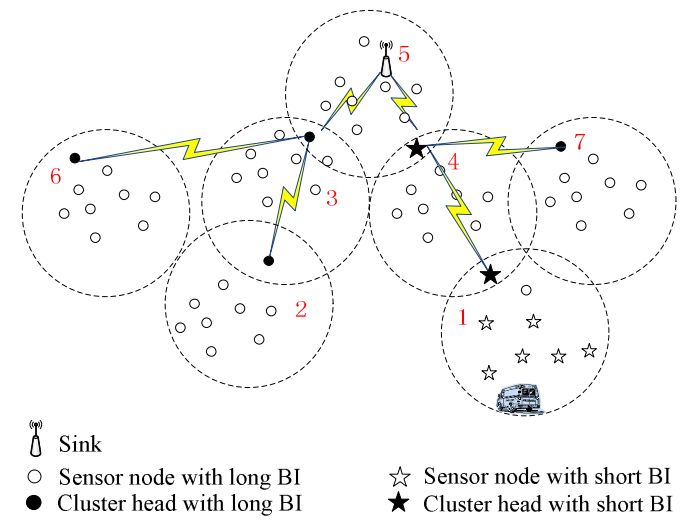

Figure 1. Network topology

The network works in a long beacon interval (BI) to save energy whenever there is no target. When a target appears, clusters that detect the target as well as the cluster heads along the data transmission path of the previous ones change to a shorter BI. The remaining clusters still keep operating with long BI as shown in Fig. 2. The SD (active interval) of different clusters in the interference range of each other should not overlap to avoid collision among different clusters [10]. Hence, an initial arrangement of the SDs is needed to avoid collisions.
Without loss of generality, we arrange the SD of different clusters as in D-MAC [11] to decrease the end-to-end delay. In this arrangement, the SD a parent cluster is arranged after the SD of its children to guarantee the uplink data to be transmitted to the sink without waiting for the inactive period of its parent. Other initial schedules of the SD are also feasible but introducing different end-to-end delay.
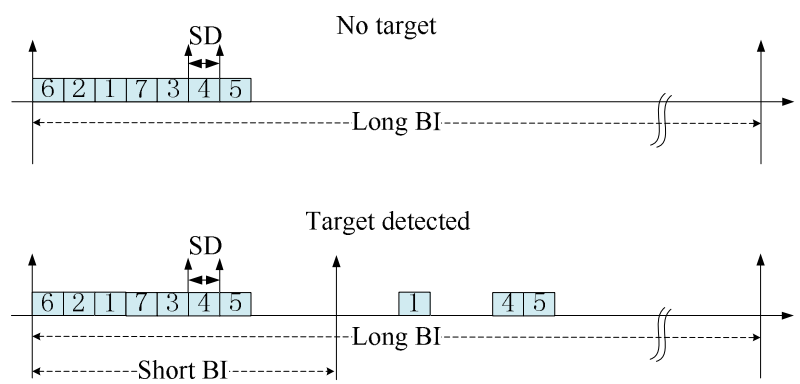

Figure 2. Dual-rate BI schedule

The dual-rate beacon scheduling method requires the sum of different clusters SD arranged during one superframe to be less than the short BI; otherwise, collision would happen due to the simultaneous transmission among different clusters. Anyway, there are optimal algorithms that can minimize the sum of SDs based on the interference metrics even when the network is very large [12]. This is out of the scope of this paper.

In dual-rate beacon scheduling, the cluster head can broadcast two types of beacons, SLOW BEACON and FAST_BEACON. When operating with long BI, only slow beacons are sent. With short BI, a number of fast beacons are sent between the slow beacons as shown in Fig. 3.

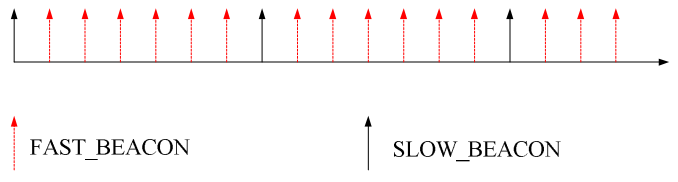

Figure 3. Beacon type sequence

\section{B. Switching Schemes}

The switching between long and short beacon intervals proposed in [9] uses four states. In this paper we consider two different switching schemes and compare their performance. The former, upon target detection, forces the node to wait in LONG_BI state until the next SD of its cluster (Fig. 4).

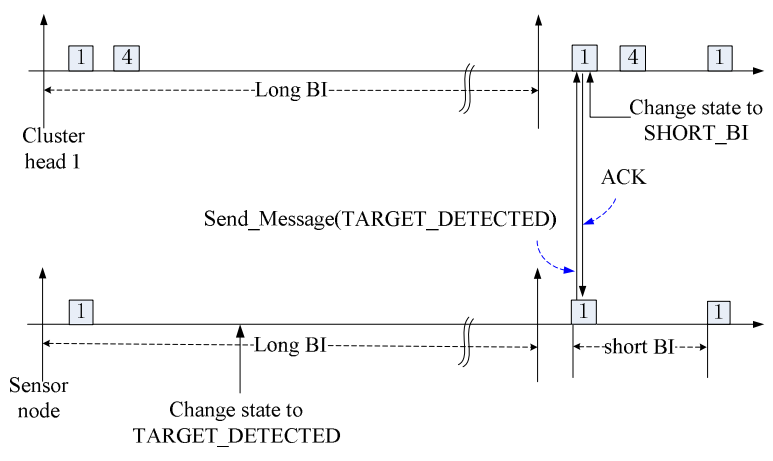

Figure 4. Slower switching scheme 
This scheme imposes a potential long BI period latency upon target detection. To reduce this initial latency we propose an enhanced rate switching scheme in which the cluster head wakes up regularly with a short BI interval and for a short period, currently for $4 \mathrm{~ms}$, to listen to possible children notifications of target detection. If there is no target, the child node will not transmit any requests and the cluster head returns to sleep. In case of target detection, the cluster head will receive the notification and change to SHORT_BI state, starting to transmit fast beacons after one short $\overline{\mathrm{BI}}$ interval (Fig. 5). Therefore, the maximum latency from target detection to SHORT_BI state is two short BI intervals.

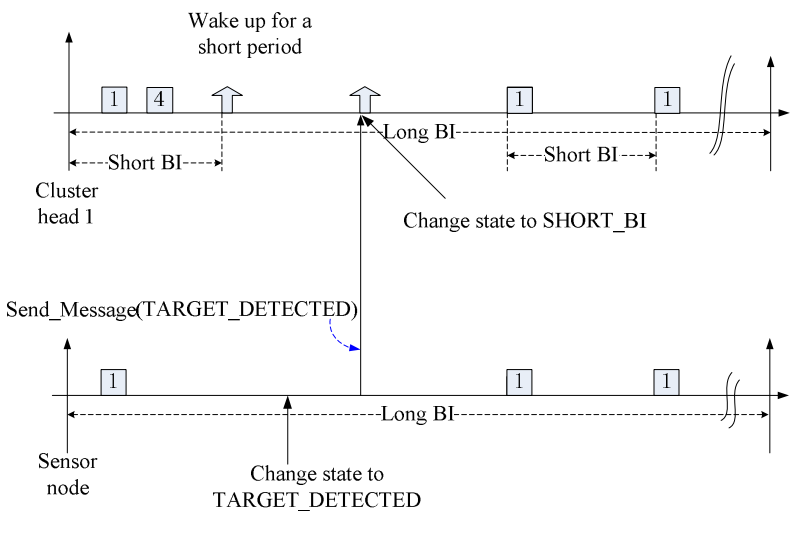

Figure 5. Enhanced switching scheme

The opposite rate switching, i.e., from short BI to long BI, is done similarly in both schemes and it is done as soon as the SLOW_BEACON is detected, which is transmitted by a cluster head when the target is no longer in range.

In these two switching schemes, we assume that the sensor node can detect the target when in the sensing range while the node processor is in sleep or idle state, which was implemented in our previous small size target tracking system [13].

\section{SimUlation Results}

In target tracking systems the network size is generally large in area and number of nodes for sensory redundancy. In [9] we studied the a case with 97 nodes. In this section we deploy 225 nodes in a $700 \mathrm{~m} \times 700 \mathrm{~m}$ area and carry out a performance study using the OPNET simulator [15]. The default simulation configuration parameters are in Table I

TABLE I. Default Settings

\begin{tabular}{|c|c|c|c|}
\hline Parameter & Value & Parameter & Value \\
\hline Long BO & 11 & Short BO & 7 \\
\hline SO & 1 & Tx_current(mA) & 21 \\
\hline Max_children & 13 & Rx_current(mA) & 23.3 \\
\hline Max_router & 5 & Idle_current(uA) & 3200 \\
\hline Max_depth & 4 & Sleep_current(uA) & 27 \\
\hline Nodes number & 225 & MAC buffer size $(\mathrm{B})$ & 1250 \\
\hline Target data size $(\mathrm{B})$ & 20 & Sensor radius $(\mathrm{m})$ & 70 \\
\hline Target data interval $(\mathrm{s})$ & 3 & Target Speed $(\mathrm{km} / \mathrm{h})$ & 30 \\
\hline
\end{tabular}

In the following simulation, SRL denotes single rate beacon working mode with long beacon interval, SRS denotes single rate beacon working mode with short beacon interval, DRBSw denotes dual-rate beacon scheduling method with normal switching scheme, EDRBSw denotes dual-rate beacon scheduling method with enhanced switch scheme. Since in [9] we have already illustrated that our method can achieve low energy consumption, hereby we focus on the average packet delay. In particular, we show the impact of three parameters, namely long beacon interval, short beacon interval and target information interval, as well as the impact of the target speed.

\section{A. Long beacon interval}

Figure 6-top shows the variation of the end-to-end delay with the long $\mathrm{BO}$ parameter. As expected, this parameter has a direct impact on SRL and DRBSw, that depend on it, and no impact on SRS and EDRBSw, which depend uniquely on the short BO. SRL and SRS impose the highest and lowest delays, respectively. The increasing delay of DRBSw with long BO is reasonable since when nodes detect a target and wake up, all the data generated waits for an initial long beacon interval until the superframe comes and the beacon interval switches to short. On the contrary, with EDRBSw nodes have the chance to notify the cluster head every short beacon interval and thus the impact of the long beacon interval can be ignored. However, the beacon interval cannot be arbitrarily long. For example, the simulations in [9] show that there is a minimum in energy consumption with $\mathrm{BO}$ between 10 and 11 .
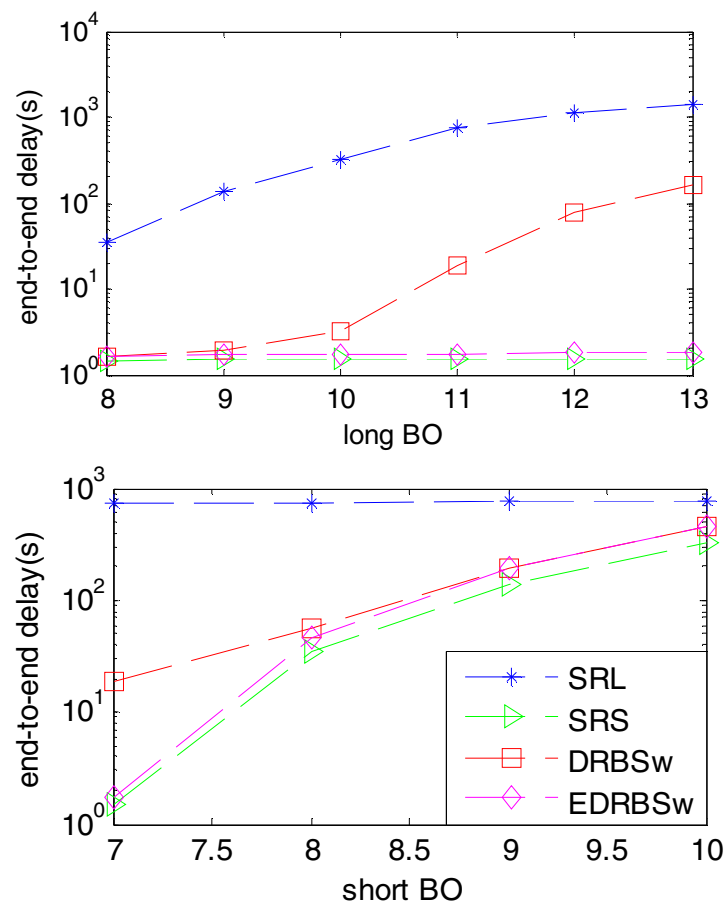

Figure 6. Impact of long BO and short BO on the end-to-end delay

\section{B. Short beacon interval}

Figure 6-bottom shows that the end-to-end delay is very sensitive to short beacon interval, except for SRL that does not depend on this parameter. Decreasing the short beacon interval has a significant impact on reducing the data propagation delay, 
which is even more pronounced with EDRBSw and SRS. Assuming that target detection periods are short with respect to the network operation time, because of the impact on energy, we can reduce this parameter as long as it is larger than the sum of the SDs of the different clusters.

\section{Target information interval}

This parameter is the period with which data on the target is sent upon target detection. The higher the better in terms of target localization accuracy but increases the traffic load. Figure 7-top shows that EDRBSw can be reduced to near $2 \mathrm{~s}$, while DRBSw has an initial delay up to one long BI, which causes larger delay as shown in Fig. 7-top.

\section{Target speed}

Figure 7-bottom shows that the target speed has almost no impact on the end-to-end delay except when using DRBSw. In this case, as the speed grows there are more nodes being awaken and trying to transmit data during the long inactive period. Thus, DRBSw is more suitable for low speed targets.
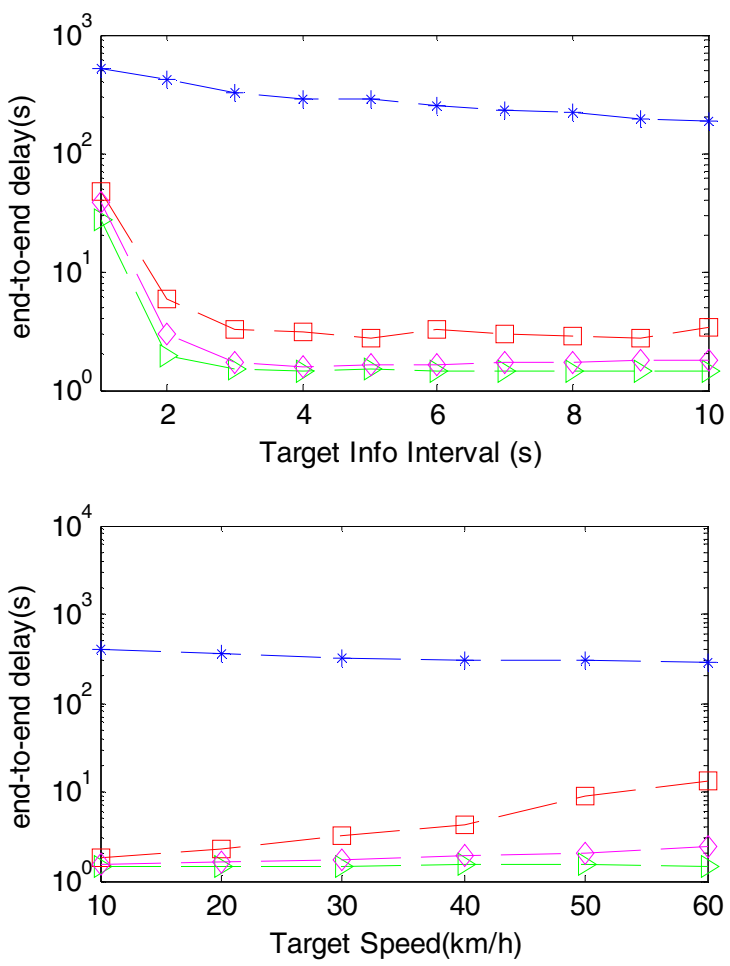

Figure 7. Impact of target information interval and target speed on the end-to-end delay (the legend is the same as in Fig. 6)

\section{EXPERIMENTAL RESULTS}

To validate the practicality of our method, we built a testbed based on CC2430 [15] network controllers and using the $\mu \mathrm{cOSII}$ operating system. Firstly, we analyze the performance of a star network in terms of different beacon interval and superframe duration. Then, we test the performance of a multicluster network using our dual-rate beacon scheduling method.

The star network consists of four end devices and one coordinator (Fig. 8). The average delay measured with different
$\mathrm{BO}$ and SO is shown in Table II while Table III shows the packet reception ratio (PRR). The delay decreases when $\mathrm{BO}$ decreases and SO increases, as expected. The packet reception ratio decreases below $100 \%$ due to saturation of the network capacity with higher BO and shorter SO. Since a cluster-tree network is composed of multiple star networks connected hierarchically, we expect a similar delay dependence.

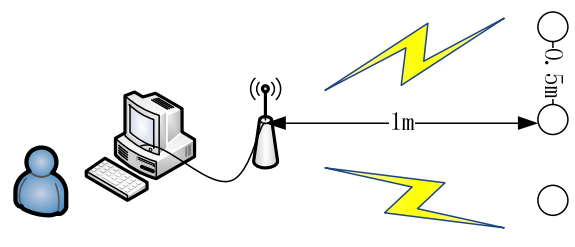

$\oiiint^{((p))} \operatorname{sink}$ Sensor node

Figure 8. Start network

TABLE II. AVERAGE DELAY (s)

\begin{tabular}{|c|l|l|l|}
\hline Delay(s) & SO=0 & SO=1 & SO=2 \\
\hline BO=7 & 2.59 & 1.07 & 1.04 \\
\hline BO=9 & 62.58 & 29.08 & 5.74 \\
\hline BO=10 & 137.85 & 68.2 & 28.5 \\
\hline
\end{tabular}

TABLE III. PACKET RECEPTION RATIO

\begin{tabular}{|c|c|c|c|}
\hline PRR & SO=0 & SO=1 & SO=2 \\
\hline BO=7 & $97.75 \%$ & $100 \%$ & $100 \%$ \\
\hline BO=9 & $41.5 \%$ & $79.75 \%$ & $100 \%$ \\
\hline BO=10 & $24 \%$ & $42.75 \%$ & $77.75 \%$ \\
\hline
\end{tabular}

Moreover, in a target tracking system, the number of the nodes that detect the target varies all the time. Therefore, we analyze the network with 6 and 8 end devices. The results are shown in table IV with $\mathrm{BO}=7$ and $\mathrm{SO}=1$. Both delay and packet reception ratio show a degradation of the network performance as the number of end devices increases. We believe that an effective use of the network with larger number of nodes requires a node selection algorithm to decrease the concurrent transmission.

\section{TABLE IV. DELAY \& PRR}

\begin{tabular}{|c|c|c|}
\hline Number of end devices & Delay(s) & PRR \\
\hline 6 & 1.09 & $83.5 \%$ \\
\hline 8 & 1.47 & $78.5 \%$ \\
\hline
\end{tabular}

Then, we implemented the dual-rate beacon scheduling method in a beacon-enabled cluster-tree ZigBee network with 31 nodes and four clusters deployed as in Figure 9.

We then grouped the nodes in 8 subgroups with three nodes each, two per cluster. With these subgroups we simulate a target passing and its detection by making the respective nodes 
start generating target information packets after a certain initial offset. The offsets are $5.5 \mathrm{~s}, 30.5 \mathrm{~s}, 55.5 \mathrm{~s}, 80.5 \mathrm{~s}, 105.5 \mathrm{~s}, 130.5 \mathrm{~s}$, $155.5 \mathrm{~s}, 180.5 \mathrm{~s}$ which correspond to a certain target speed. Each node that detects the target generates 20 packets containing 12 Bytes of information with a time interval of $3 \mathrm{~s}$. The default long beacon order and short beacon order are set to 10 and 6 , and the superframe order is set to 2 . In the implementation of EDRBSw we also have to consider the short wakeup time every short beacon interval, which was $4 \mathrm{~ms}$ in the simulations. However, in the actual implementation we have to consider the clock drift between parent and child that can occur during the superframe, despite the synchronization in the beginning when receiving the beacon frame from the parent. Therefore, we use $10 \mathrm{~ms}$ of wake up time to guarantee that the parent can receive the potential indication packets from its children.

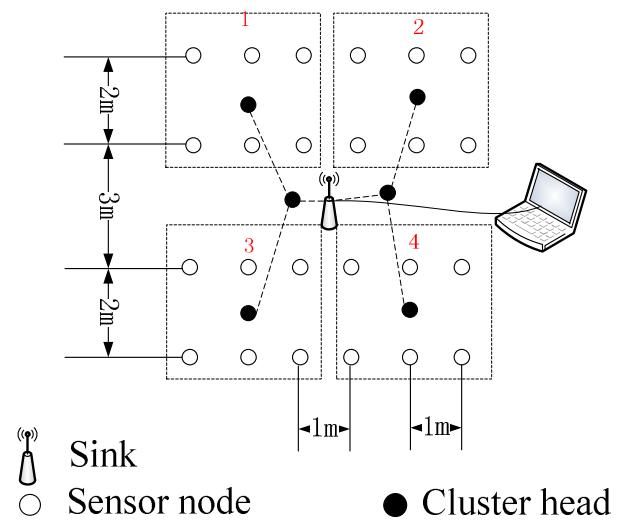

Figure 9. Network topology used in the experiments

The average delays and packet reception ratios are shown in table $\mathrm{V}$ while the individual packet delays are shown in Fig. 10.

TABLE V. DELAY \& PRR

\begin{tabular}{|c|c|c|}
\hline Method & Delay(s) & PRR \\
\hline SRL & 40.6 & $30.83 \%$ \\
\hline SRS & 0.906 & $92.42 \%$ \\
\hline DRBSw & 1.21 & $87.92 \%$ \\
\hline EDRBSw & 0.965 & $92.08 \%$ \\
\hline
\end{tabular}

The experimental results are consistent with the simulation. Generally, from the results we see that our methods exhibit better performance than the original ZigBee network configured with a long beacon interval. On the other hand, our methods still present an energy consumption close to that of this mode [9]. In Figure 10, the spikes that occur with DRBSw and EDRBSw are due to target transitions from cluster to cluster. Note that when the target moves between two subgroups in the same cluster, there is no extra latency since the cluster head has already been notified by the other subgroup and is already operating with the short beacon interval.

In the MAC layer we use a queue of ten packets to buffer the data. In SRL, packets are accumulated in the buffer due to the long inactive period but the buffer is not enough and a certain number of packets are dropped meaning that the capacity of the network was exhausted. So the PRR is very low for SRL. The overall PRR is affected by the CSMA/CA algorithm in the MAC of ZigBee. In this test we ignore the possibility of data fusion in the cluster head, we simply forward all packets leading to higher traffic volumes. Thus, the closer to the coordinator, the higher the probability of packet collisions and packet losses. As a further consideration, PRR could be raised by adopting suitable data fusion algorithm.
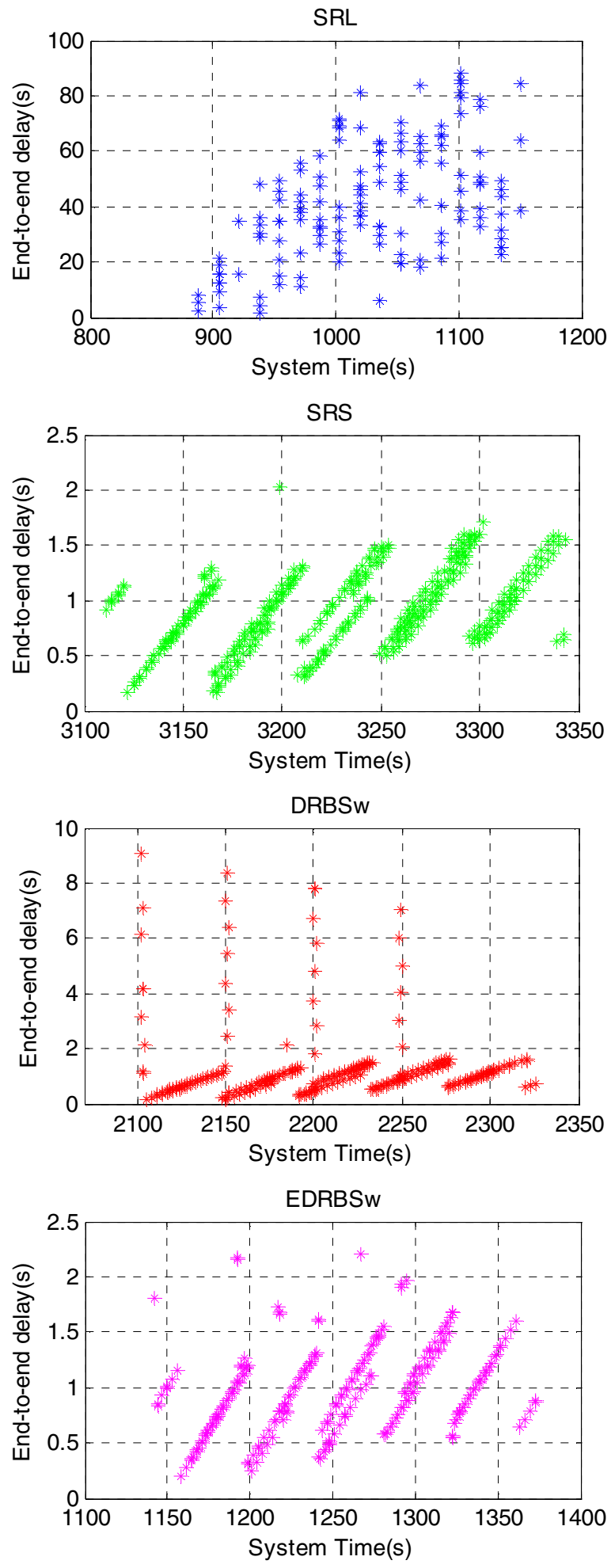

Figure 10. End-to-end delay with different beacon rates 
Finally, we also carried out an indirect test on energy consumption of the ZigBee network. We assessed the energy level of an end device with $\mathrm{BO}=10$ and 6 for several days by measuring the respective voltage level (Fig. 11). The node enters sleep mode if there is no packet in the buffer after receiving the beacon frame or after finishing sending the previous packet. The difference in voltage drop is substantial showing the impact of the longer sleeping periods of a long beacon interval. Considering the major part of the ZigBee network to be composed of end devices and the fact that targets appear occasionally and excite a few nodes at a time, the energy consumption of our schemes, both DRBSw and EDRBSw, is close to that of a network operating with a fixed long BO.

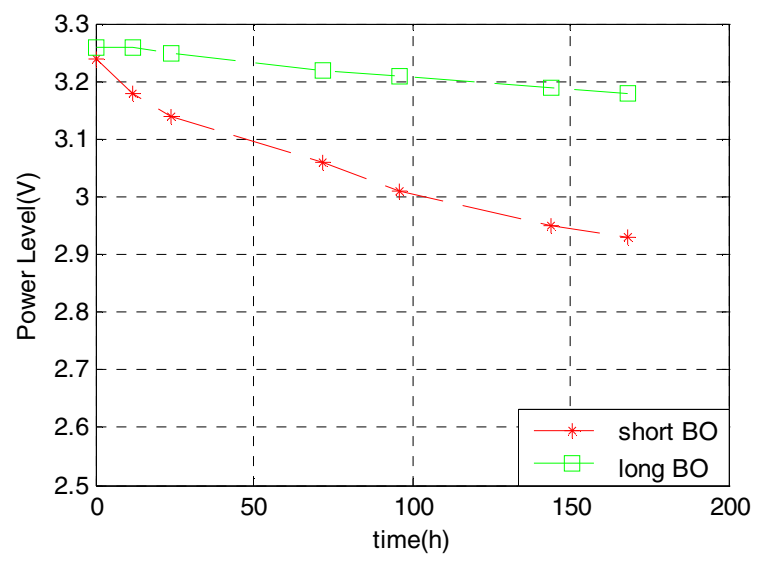

Figure 11. Battery voltage level of end devices

\section{CONCLUSION}

This paper analyzed the performance of a dual-rate beacon scheduling method for ZigBee/IEEE 802.15.4 for target tracking systems, which can be of high relevance within the scope of CPNS. This method was developed to provide a good trade-off between energy consumption and transmission delay. In this paper we carried out an analysis of such method, using simulation, regarding the impact of different configuration parameters and of the target speed on the transmission delay. Moreover, we experimented with a test-bed that implemented the dual-rate beacon scheduling method. The experimental results agree with the expectations, showing that short transmission delays can be achieved while the network is operating with a long beacon interval most of the time, thus with low energy consumption.

\section{REFERENCES}

[1] E. A. Lee. Cyber physical systems: Design challenges. Technical Report UCB/EECS-2008-8, EECS Department, University of California, Berkeley, January 2008

[2] ZigBee Specification 2006.

[3] IEEE 802.15.4 Standard.

[4] M. Ferrari, L. Pizziniaco, "An adaptive scheme for active periods schedule in IEEE 802.15.4 wireless networks," in Proc. 3rd International Symposium on Wireless Communication Systems(ISWCS '06), vol., no., pp.665-670, 6-8 Sept. 2006.

[5] J. Lee, J. Y. Ha, J. Jeon, D. S. Kim, W. H. Kwon, "ECAP: A bursty traffic adaptation algorithm for IEEE 802.15.4 Beacon-Enabled networks," in Proc. IEEE 65th Vehicular Technology Conference (VTC2007-Spring), vol., no., pp.203-207, 22-25 April 2007.

[6] J. Lim, B. T. Jang, "Dynamic duty cycle adaptation to Real-Time data in IEEE 802.15.4 based WSN," in Proc. 5th IEEE Consumer Communications and Networking Conference(CCNC 2008), vol., no., pp.353-357, 10-12 Jan. 2008.

[7] M. Neugebauer, J. Plönnigs, K. Kabitzsch, "A new beacon order adaptation algorithm for IEEE 802.15.4 networks," in Proc. the Second European Workshop on Wireless Sensor Networks, vol., no., pp.302311, 2005.

[8] M. Neugebauer, J. Plönnigs, K. Kabitzsch, "Duty cycle adaptation with respect to traffic," in Proc. 10th IEEE Conference on Emerging Technologies and Factory Automation(ETFA 2005), vol.2, no., pp.8 pp.432, 19-22 Sept. 2005.

[9] S. Chen, L. Almeida, Z. Wang, "A dynamic dual-rate beacon scheduling method of ZigBee/IEEE 802.15.4 for target tracking," The 6th International Conference on Mobile Ad-hoc and Sensor Networks (MSN'10), vol., no., pp.103-109, 20-22 Dec 2010

[10] A. Koubaa, M. Alves, M. Attia, and A. V. Nieuwenhuyse, "Collisionfree beacon scheduling mechanisms for IEEE 802.15.4 / ZigBee clustertree wireless sensor networks," in Proc. 7 th International Workshop on Applications and Services in Wireless Networks (ASWN2007), May 2007.

[11] G. Lu, B. Krishnamachari, and C. Raghavendra. "An adaptive energyefficient and low-latency mac for data gathering in sensor networks," in Proc. 18th International Parallel and Distributed Processing Symposium(IPDPS2004), vol., no., pp. 224, 26-30 April 2004.

[12] R. Burda, C. Wietfeld, "A distributed and autonomous beacon scheduling algorithm for IEEE 802.15.4/ZigBee networks," in Proc. IEEE International Conference on Mobile Ad-hoc and Sensor Systems (MSN2007), MASS 2007, vol., no., pp.1-6, 8-11 Oct. 2007.

[13] J. Luo, D. Feng, S. Chen, P. Zhang, M. Bao, Z. Wang. "Experiments for on-line bearing-only target localization in acoustic array sensor networks," in Proc. 8th World Congress on Intelligent Control and Automation(WCICA2010), vol., no., pp.1425-1428, 7-9 July 2010

[14] M. Kohvakka, M. Kuorilehto, M. Hannikainen, T.D. Hamalainen, "Performance analysis of IEEE 802.15.4 and ZigBee for Large-Scale wireless sensor network applications", in 3rd ACM International Workshop on Performance Evaluation of Wireless Ad Hoc, Sensor, and Ubiquitous Networks (ACM PE-WASUN 2006), pp. 48-57, 2006.

[15] CC2430 datasheet

[16] www.opnet.com 